\title{
Research on Modular Construction of Gene Regulatory Network
}

\author{
Ming Zheng ${ }^{1,2, a}$, Mugui Zhuo ${ }^{1, b}$ and Guixia Liu ${ }^{2, c^{*}}$ \\ ${ }^{1}$ Guangxi Colleges and Universities Key Laboratory of Professional Software Technology, Wuzhou \\ University, Wuzhou, China \\ ${ }^{2}$ College of computer science and technology, Jilin University, Changchun 130012, China \\ a370505375@qq.com, ${ }^{b} 756456050 @ q q . c o m,{ }^{c}$ liugx@jlu.edu.cn
}

*The corresponding author

Keywords: Gene regulatory network; Network motif; Network construction; Modular construction

\begin{abstract}
Gene regulatory network is a large number of genes regulated by transcriptional factors and translated into protein complex information so as to realize the biological function, is an important part of people's understanding of biological processes and gene function. In order to understand the regulatory mechanism of gene regulatory networks, the network topology and its organization is one of the most important research contents. It can not only describe the local characteristics of the network, but also can reveal the construction method of the control network. The control network can be divided into 4 layers: control elements, Motif, modules and the whole network. At present, more and more people have recognized the hierarchy. Motif and two key module is discussed in this article are compared and analyzed in recent years on many aspects of the research content of network organization structure, expounds the biological significance of various research results and conclusion have, and pointed out the existing problems. On this basis, the paper also puts forward the possible research direction for these problems, and looks forward to the prospect of modular organization of gene regulatory network.
\end{abstract}

\section{Introduction}

Regulation is an important mechanism of life activities, and plays an important role in the process of life. However, the regulation of relationship between cells is very complicated, even in simple model organisms (such as E.coli[1] and eukaryotic yeast[2]) are regulatory interactions of tens of thousands of. High throughput technologies (such as chromatin immunoprecipitation chip technology, Chromatin Immunoprecipitation-chip, ChIP- chip) development, to control the emergence of a large number of data, so that people on the regulatory network composed of various regulatory relationships from the whole possible[3].

The study of a complex system often begins with its organizational structure. At present, it is far from fully understanding the regulatory mechanisms involved in gene regulatory networks[4]. Therefore, a large number of systems biology methods are studied from the aspects of network structure, organization and functional analysis. It has been successfully used to describe the state of the disease and to classify cancer by using a regulatory network topology[5]. In the aspect of network organization, the control network can be divided into four layers: element (regulatory), Motif, Module and the whole network. Among them, divided into cis regulatory elements (cis-) and trans (trans-) two types, respectively, by combining gene sequence and transcription factor binding sites of DNA in the sequence of gene activation or inhibition; Motif and module are regulation from a set of genes, is an important analysis the structure of local features of the network and network composition and Study on the regulation mechanism of the. This paper studies on the gene regulatory network organization from two aspects and Motif module are summarized, and further explore the genetic regulatory network research and regulatory mechanism of problems in the analysis of gene regulatory network modular organization of the future possible development direction. 


\section{Motif of Gene Regulatory Networks}

Motif is a simple network structure unit has a network[6], it is said that the small scale model of specific regulatory interaction between transcription factors and target genes, usually by a few genes according to a certain topological structure form. For example, there are 3 kinds of regulation network of Escherichia coli motif: (Feed Forward Loop, feed-forward loop FFL, motif (Single) Input single input Motif, SIM) and control (Dense Overlapping dense overlapping Regulon, DOR)[7]; and for the beer yeast transcriptional regulatory network, people put forward 6 kinds of network motif. They are: self-regulation (Auto- regulation), multi component circuit (Multi-Component Loop), feed forward loop, single input motif, multi input motif (Multi-Input Motif) and Chain (Regulator). These 6 kinds of motif are widely used in biological networks, they are some of the structural characteristics of the model, is the basic unit of complex network structure, as shown in fig.1:

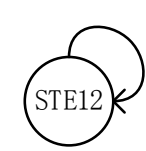

Auto-regulation

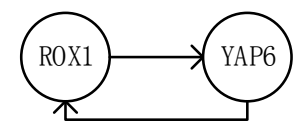

Multi-component loop

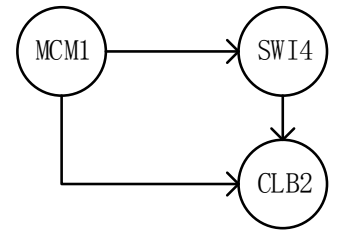

Feedback loop

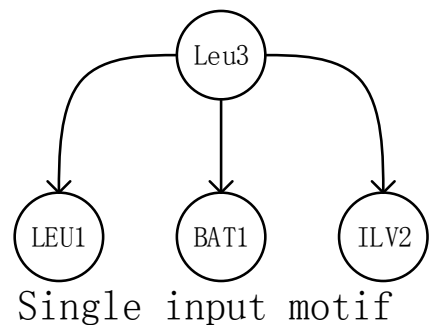

Single input motif

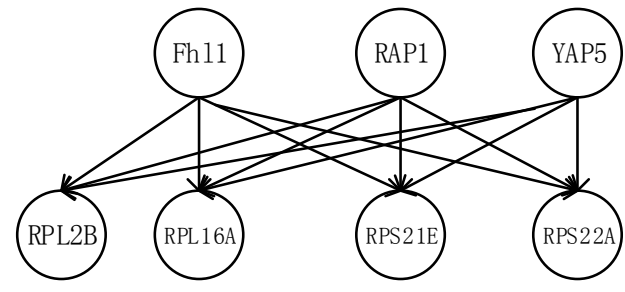

Multi-input motif

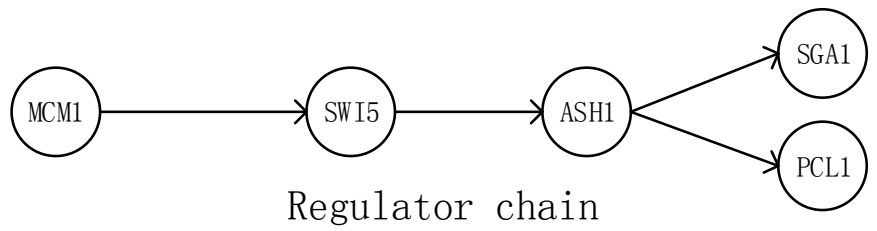

Figure 1. Six types of motifs of yeast transcriptional regulatory network

Different motif showed different conduction modes. For example: transcriptional regulation network of Escherichia coli without feedback loop, it also shows that the relative simplicity of gene regulation mechanism. For eukaryotes, feedback is an important mechanism, which plays an important role in the process of life. Recent studies show that the negative feedback not only speed up the response time of gene pathway, and can reduce the difference of protein level in each cell, and positive feedback from the opposite, it slows down the response time of the gene pathway, the differences between the cells increased. Based on the relationship between single species regulation (activation and suppression of two), a feed-forward loop can be divided into consistent and inconsistent two. Among them, the uniform feedforward loop motif can be regarded as a signal sensitive delay element in the transcription network, and the first kind of inconsistent feedforward loop can generate a pulse signal and accelerate the system response.

The analysis of kinetic characteristics of Motif is an important aspect of understanding the mechanism of regulation. In the regulatory network that contains thousands of biochemical reactions, Motif is actually a subset of functional and structural dynamics. Grammaticos[8] was used to analyze the dynamics of the regulatory chain, and the $S$ function was used to give the equation. It is worth noting that the proposed method can also be extended to the dynamic analysis of the motif module. A large 
number of gene regulatory networks routing motif. A single motif can reveal many details of the network features or content, is the basic unit of gene regulatory network. The relationship between these basic units and the way to form a network by these basic units are more and more concerned by people.

\section{Gene Regulatory Network Module}

From the above analysis, we can see that Motif is a small-scale model with structural features, although it can give some explanation for the mechanism of biological process regulation. The study shows that the module of the dynamic characteristics and the biological function of motif with the appropriate scale is composed of a plurality of. At present, people have done some research on the method of module partition and function correlation analysis. According to the definition of the module, we will gene regulatory networks are divided into three categories: transcription module, data obtained in some biological conditions or biological processes in dynamic control sub network and control network based on function module based on topology of gene chip.

Module Based on Gene Expression Data. In recent years, gene expression microarray data[9] are often used to analyze the relationship between transcription factors and target genes. Under different biological processes or environmental conditions, the changes of gene expression can be carried out through the regulation relationship, and finally the biological functions can be realized. Therefore, the relationship between the expression of multiple genes in time series data has become an important information for understanding the regulation mechanism of biological processes.

Conditional Specific Regulatory Networks. The data of gene regulation include biological environment information. In recent years, it has been found that the gene regulatory network is a dynamic process[10]. Therefore, the analysis of the dynamic characteristics of the network has become a method of mining regulation mechanism and regulation of network organization. The more accurate regulation of dynamic network representation is more advantageous to the modular organization of the regulatory network. On the one hand, the dynamic network itself can be used as a function of the gene regulatory network and biological processes related to the interpretation module, it shows that certain biological processes in a certain environmental conditions with time changes in the regulatory process. On the other hand, compared with the dynamic network under many conditions, we can make people more clear about the mechanism of biological process. Compared with static network, dynamic network can better reflect the relationship between genes. In addition to the regulation of the relationship between the information, the dynamic control network also gives the existence of the regulatory relationship between the conditions and time.

Functional Module based on network topology. Although the above two modules can reveal the modular organization of gene regulatory networks, most of them use information other than the number of regulatory networks, such as gene expression data, transcription factor binding site data, etc.. At present, it is controversial whether the network data can be used to explain the biological significance. Since the discovery of the scale-free characteristics of complex networks, a large number of studies have been carried out to explain some of the biological functions from the topological structure. Although the network topology cannot play a decisive role in biological function, it contains a lot of information related to biological function. The network topology is a very important information for the study of the organization mode of the network function module.

Correlation Analysis of Functional Modules. Module partition is an important research method for people to understand complex systems. For biological systems, it is generally believed that a variety of genes or proteins that perform the same function tend to form modules. Therefore, the analysis of the functional modules can make people understand the biological processes and understand the biological structure and organization. From the above we can see that the dynamic network provides more accurate information about the regulation of gene expression. Therefore, the analysis of the dynamics of the network will be more effective in explaining the process of cell regulation. Therefore, it is also pointed out that the more accurate and detailed module becomes an important research process of dynamic analysis. Finally, the biological networks are not isolated from each other, there is a very close relationship between each other. The analysis of the functions of a variety of biological networks will 
provide a wider range of information for exploring the ways and means of achieving the function of life processes.

\section{Conclusion}

It is of great theoretical and practical significance to analyze and find the way to organize the network. Through the research on the direction of the people will be more clearly and accurately understand the regulation mechanism of regulation pathway, rational division has a good explanation of the function module, on the basis of in-depth and comprehensive understanding of the function of each module and function on the understanding of biological processes, to explore the mysteries of life.

\section{Acknowledgements}

This work was supported by grants from The National Natural Science Foundation of Chi-na (No. 61502343, No. 61373051, and No. 61402423), China Postdoctoral Science Foun-dation funded(No. 2016M590260), the Guangxi Natural Science Foundation (No. 2015GXNSFBA139262), the Science Research Funds for the Guangxi Universities (No. KY2015ZD122), Guangxi Colleges and Universities Key Laboratory of Professional Software Technology, Wuzhou University.

\section{References}

[1] Cho, B.K., Federowicz, S.A., Embree, M., Park, Y.S., Kim, D., and Palsson, B.O.: 'The PurR regulon in Escherichia coli K-12 MG1655', Nucleic Acids Research, 2011, 39, (15), pp. 6456-6464

[2] Lelandais, G., Goudot, C., and Devaux, F.: 'The evolution of gene expression regulatory networks in yeasts', Comptes Rendus Biologies, 2011, 334, (8-9), pp. 655-661

[3] Chai, L.E., Loh, S.K., Low, S.T., Mohamad, M.S., Denis, S., and Zakaria, Z.: 'A review on the computational approaches for gene regulatory network construction', Computers in Biology and Medicine, 2014, 48, pp. 55-65

[4] Hernando, C.E., Romanowski, A., and Yanovsky, M.J.: 'Transcriptional and post-transcriptional control of the plant circadian gene regulatory network', Biochimica et Biophysica Acta-Gene Regulatory Mechanisms, 2017, 1860, (1), pp. 84-94

[5] Chatterjee, S., Kapoor, A., Akiyama, J.A., Auer, D.R., Lee, D., Gabriel, S., Berrios, C., Pennacchio, L.A., and Chakravarti, A.: 'Enhancer Variants Synergistically Drive Dysfunction of a Gene Regulatory Network In Hirschsprung Disease', Cell, 2016, 167, (2), pp. 355-+

[6] Babu, M.M.: 'Structure, evolution and dynamics of transcriptional regulatory networks', Biochemical Society Transactions, 2010, 38, pp. 1155-1178

[7] Camas, F.M., and Poyatos, J.F.: 'What Determines the Assembly of Transcriptional Network Motifs in Escherichia coli?', Plos One, 2008, 3, (11)

[8] Grammaticos, B., Carstea, A.S., and Ramani, A.: 'On the dynamics of a gene regulatory network', Journal of Physics a-Mathematical and General, 2006, 39, (12), pp. 2965-2971

[9] Li, H.Y., Zhao, X.N., Wang, J., Zong, M.R., and Yang, H.L.: 'Bioinformatics analysis of gene expression profile data to screen key genes involved in pulmonary sarcoidosis', Gene, 2017, 596, pp. 98-104 
[10] Goode, D.K., Obier, N., Vijayabaskar, M.S., Lie-A-Ling, M., Lilly, A.J., Hannah, R., Lichtinger, M., Batta, K., Florkowska, M., Patel, R., Challinor, M., Wallace, K., Gilmour, J., Assi, S.A., Cauchy, P., Hoogenkamp, M., Westhead, D.R., Lacaud, G., Kouskoff, V., Gottgens, B., and Bonifer, C.: 'Dynamic Gene Regulatory Networks Drive Hematopoietic Specification and Differentiation', Developmental Cell, 2016, 36, (5), pp. 572-587 